\section{PATTERN APPROACH TO INTERSTITIAL LUNG DISEASE IN HRCT: A PICTORIAL CASE SERIES}

\author{
SUMANTA ROY ${ }^{\mathrm{a}}$, M. GOPINATH ${ }^{\mathrm{b}}$, P. SHARMA ${ }^{\mathrm{c}}$ AND R. RAVI KUMAR ${ }^{\mathrm{d} 1}$ \\ ${ }^{\mathrm{a}} \mathrm{Gd}$ Spl. (Rad) Department of Radiology, $164 \mathrm{MH}$, India \\ ${ }^{\mathrm{bc}} \mathrm{Sr}$. Adv. (Rad) Department of Radiology, CHEC, Kolkata, India \\ ${ }^{\mathrm{d} C}$ Consultant (Rad) Department of Radiology, CHEC, Kolkata, India
}

\begin{abstract}
The term Interstitial Lung Disease (ILD)comprises a heterogeneous group of disorders of known and unknown etiology, characterized by dyspnea, diffuse parenchymal lung abnormalities, restrictive pulmonary function and impaired gas exchange. Although ILDs are more commonly seen in adults, some forms such as hypersensitivity pneumonia and LCH are encountered in children as well. In 2001, the American Thoracic Society (ATS) and European Respiratory Society (ERS) standardized the terminology for Idiopathic Interstitial Pneumonias (IIPs).The recent update of this classification separates the IIPs into four groups: chronic fibrosing IIPs, acute or sub-acute IIPs, smoking related IIPs and rare IIPs. The wide variety of ILDs and concurrent non-specific clinical symptomatology, poses a diagnostic challenge to the radiologist. This article illustrates a systematic and pattern approach to HRCT interpretation with the aim of narrowing down the differential diagnosis in a specific clinical scenario, thereby facilitating decision making and patient management.
\end{abstract}

KEYWORDS: HRCT, Pulmonary Fibrosis, Interstitial Lung Disease

\section{Predominant HRCT Pattern}

The first step in the approach is for the radiologist to identify the dominant pattern(s) and describe it using the standard radiological terms (Hansell et al., 2008). These include reticular, nodular or reticulonodular patterns. Thickening of interstitial network of lung by fluid or fibrous tissue or infiltration by cells or other substances giving rise to reticular pattern. Thickening may be smooth as in case of interstitial pulmonary edema, or nodular is in sarcoidosis/ lymphangitis carcinomatosis or irregular as in pulmonary fibrosis. Nodules are ill defined opacities of soft-tissue attenuation. Some nodules can be of groundglass opacity. There may be air-space nodule or interstitial nodule. Depending on the size criteria, these may be micro-nodule $(<3 \mathrm{~mm})$, small $(<1 \mathrm{~cm})$ orlarge $(>1 \mathrm{~cm})$. Often both reticular and nodular patterns are seen to co-exist.

\section{Predominant Spatial Distribution of Disease}

Some ILDs tend to preferentially involve the upper lobes, where some others affect the mid or lower lobes. Therefore when attempting to reach a differential diagnosis using HRCT, the overall distribution of pulmonary abnormalities should be considered along with their morphology. Diseases associated with reticular opacities on HRCT, that can have a central or peri-hilar predominance include end stage sarcoidosis, silicosis, fibrotic NSIP, Chronic HP and Lymphangitic spread of carcinoma. A peripheral, cortical or sub-pleural predominance of abnormalities is typical of UIP and has been reported in nearly all patients with asbestosis, IPF, scleroderma, rheumatoid lung disease or other collagen vascular disease. Disease that can result in reticular opacities and have been recognized to have upper lobe predominance on HRCT include sarcoidosis, silicosis and CWP. A basal distribution is most typical of UIP, IPF, collagen vascular diseases such as rheumatoid lung disease and scleroderma. The basal predominance is also typical of fibrotic NSIP.

In addition to zonal distribution, the pulmonary nodules can also be of diagnostic value. This is particularly true for micro-nodular diseases in which the allocation of the nodules to one of the three distribution types (centri-lobular, peri-lymphatic, random) is of use to narrow the differential diagnoses.

\section{Salient Clinical Features}

Although patient history and clinical findings can be of great value in suspecting or diagnosing a specific disease, in practice, many HRCT studies are performed and interpreted with little or no relevant clinical information available. Such basic history as whether the patient's symptoms are acute or chronic, or 
whether fever is present, can be immensely helpful. For example an acute presentation with ground glass opacity narrows the differential diagnosis to acute interstitial pneumonia, diffuse alveolar hemorrhage, fulminant organizing pneumonia or atypical pneumonitis, where as ground glass opacity with progressive or chronic symptoms may include hypersensitivity pneumonitis (HP), eosinophilic lung disease, alveolar proteiniosis, RB - ILD.

Pleuritic chest pain is often associated with rheumatological diseases. Co-existent pneumothorax may be indicative of underlying cystic lung disease, especially LCH and LAM. Systemic symptoms like fever, malaise, fatigue, arthralgia may present in sarcoidosis and rheumatological diseases.

A definite history of cigarette smoking is present casesof LCH, Desquamative Interstitial Pneumonia (DIP) and Respiratory Bronchiolitis ILD.

Reliable occupational history and environmental exposure to asbestos with inspiratory crackles at the posterior lung base, not cleared by coughing may be indicative of underlying asbestosis. Other relevant information includes foreign travel which may raise the possibility of parasitic infection as an explanation of pulmonary eosinophilia.

Any Associated Findings in HRCT (Desai et al., 2019)

Additional HRCT features may suggest or exclude certain diagnoses. Thus inclusion of any of the following may be of diagnostic value in specific cases:

1. There may be pleural thickening/effusion/plaques/ Calcification which may indicate asbestos related lung disease as well as negate the possibility of IPF as a possible cause of lung fibrosis.

2. Lymphnodal enlargement (Hilar/ Mediastinal)reactive intra-thoracic nodal enlargement is a recognized "normal" in fibrotic ILDs. However, symmetrical hilar nodal enlargement may suggest a diagnosis of sarcoidosis.

3. Bronchiectasis - Patient having established pulmonary fibrosis, co-existent airway disease in a patient may point to a diagnosis of an underlying connective tissue disease, such as rheumatoid arthritis.

4. HRCT depicting non-specific interstitial pneumonia pattern with an added finding of dilation of the esophagus may points toward scleroderma as the underlying disease.

\section{Salient Distribution Patterns}

Table 1a:

\begin{tabular}{|l|l|}
\hline \multicolumn{1}{|c|}{ Disease } & \multicolumn{1}{c|}{ Findings } \\
\hline Posterior lung & \\
\hline Usual interstitial pneumonia; Nonspecific interstitial pneumonia & Fibrosis; ground-glass opacity \\
\hline Asbestosis & Fibrosis \\
\hline Sarcoidosis & Nodules; conglomerate masses \\
\hline Acute respiratory distress syndrome (ARDS) & Ground-glass opacity; consolidation \\
\hline Hypersensitivity pneumonitis & Ground-glass opacity; nodules; fibrosis \\
\hline Anterior lung & \\
\hline Post-ARDS fibrosis & $\begin{array}{l}\text { Sub-pleural fibrosis; Honeycombing; } \\
\text { Traction bronchiectasis }\end{array}$ \\
\hline
\end{tabular}

Table 1b:

\begin{tabular}{|l|l|}
\hline \multicolumn{1}{|c|}{ Disease } & \multicolumn{1}{c|}{ Findings } \\
\hline Central lung & \\
\hline Sarcoidosis & $\begin{array}{l}\text { Peri-bronchovascular nodules; conglomerate fibrosis } \\
\text { Traction bronchiectasis }\end{array}$ \\
\hline Lymphangitic spread of carcinoma & Peribroncho-vascular interstitial thickening or nodules \\
\hline Large airways diseases & Bronchiectasis (e.g., cystic fibrosis) \\
\hline Peripheral lung & \\
\hline Usual interstitial pneumonitis; Idiopathic pulmonary & Sub-pleural fibrosis; honeycombing; \\
\hline
\end{tabular}


ROY ET AL.: PATTERN APPROACH TO INTERSTITIAL LUNG DISEASE IN HRCT: A PICTORIAL CASE SERIES

\begin{tabular}{|l|l|}
\hline fibrosis; Collagen diseases; Asbestosis & sometimes ground-glass opacity \\
\hline Nonspecific interstitial pneumonia & $\begin{array}{l}\text { Sub-pleural ground-glass opacity; reticulation; } \\
\text { Sub-pleural sparing in 50\% }\end{array}$ \\
\hline Bronchiolitis obliterans organizing pneumonia & Sub-pleural consolidation or ground-glass opacity \\
\hline Desquamative interstitial pneumonia & Peripheral ground-glass opacity \\
\hline Hypersensitivity Pneumonitis & Peripheral ground-glass opacity \\
\hline
\end{tabular}

\section{Fibrotic Vs Inflammatory}

A key step in the approach is to differentiate between predominantly fibrotic and predominantly inflammatory disease. As fibrosis develops, a fine reticular pattern appears that may be diffuse. As it progresses, the reticular pattern becomes coarser with progressive loss of lung volume. Concurrent anatomical distortion and extensive honeycombing ensue that reflects end stage fibrosis. In contrast, ground glass attenuation is considered to represent active inflammation, but this is generally true only in the absence of admixed reticular abnormalities or traction bronchiectasis. It often indicates the presence of an ongoing active and potentially treatable process. For example, in patients with AIDS and acute respiratory distress, ground glass opacity visible on HRCT accurately predicts the presence of Pneumocystis jiroveci pneumonia.

Now if the disease is fibrotic by nature, the next step to address is whether it is typical of IPF. As stated in the recent ATS/ERS recommendation, IPF can be diagnosed confidently on HRCT when there is honeycombing with little ground glass attenuation in a predominantly basal and sub-pleural distribution. It is now known that HRCT appearances considered typical of IPF have a positive predictive value of over 95\% (Hunninghake et al., 2001). In fact, honeycombing appears to be the most useful HRCT feature to differentiate usual interstitial pneumonia (UIP) from either cellular or fibrotic NSIP.

\section{SPECTRUM OF ILD}

\section{Acute Interstitial Pneumonitis}

It is the only entity among the ILDs, with acute onset of symptoms which progresses rapidly (Vourlekis et al., 2000). AIP represents an idiopathic disease, which occurs equally in men and women with a mean age of 50 yrs. It usually occurs in previously healthy people, with an antecedent viral like illness, such as myalgia, arthralgia and fever. Smoking might not increase the risk of developing AIP.
Most patients develop severe dysponea within 03 weeks of the onset of symptoms and seek medical attention with signs of pneumonic consolidation with diffuse crackles (Fergusion and Berkowitz, 2012). A restrictive pattern with reduced diffusing capacity is typical in pulmonary function tests. The condition rapidly progresses to acute respiratory failure, which requires mechanical ventilation with oxygen supplementation.

Ground glass attenuation resulting from the presence of alveolar septal edema and hyaline membrane. There is air-space consolidation in dependent areas of lung, reflecting the intra-alveolar edema and hemorrhage. These features are seen in the exudative phase. These signs are usually bi-basilar but often can be diffuse or involve upper lobes. Consolidations, caused by intraalveolar fibrosis are seen in the organizing phase, characterized by lung architectural distortion, traction bronchiectasis and cysts (Primack et al., 1993).

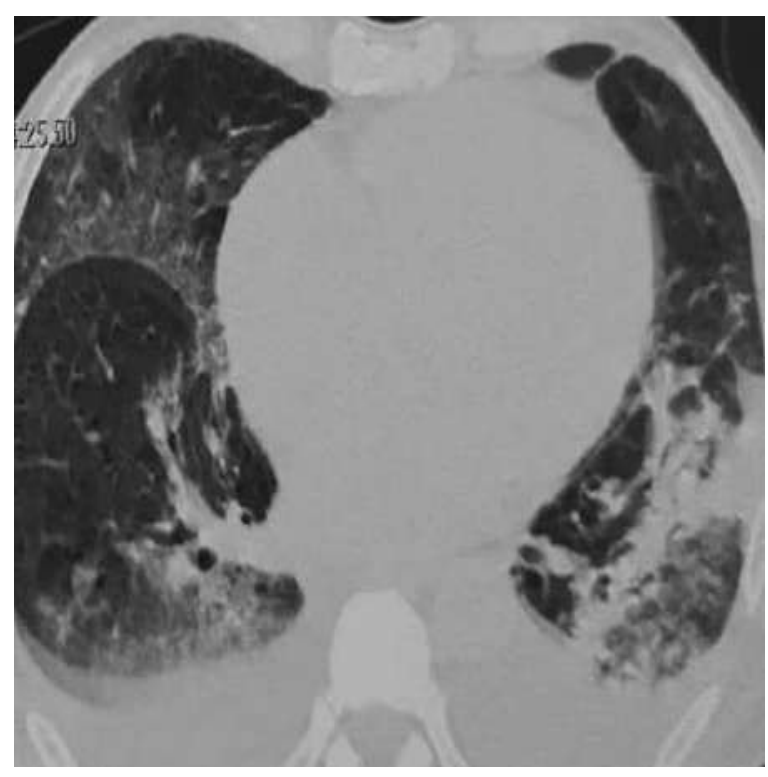

Figure 1a: Peri-bronchial consolidation and bilateral pleural effusion 


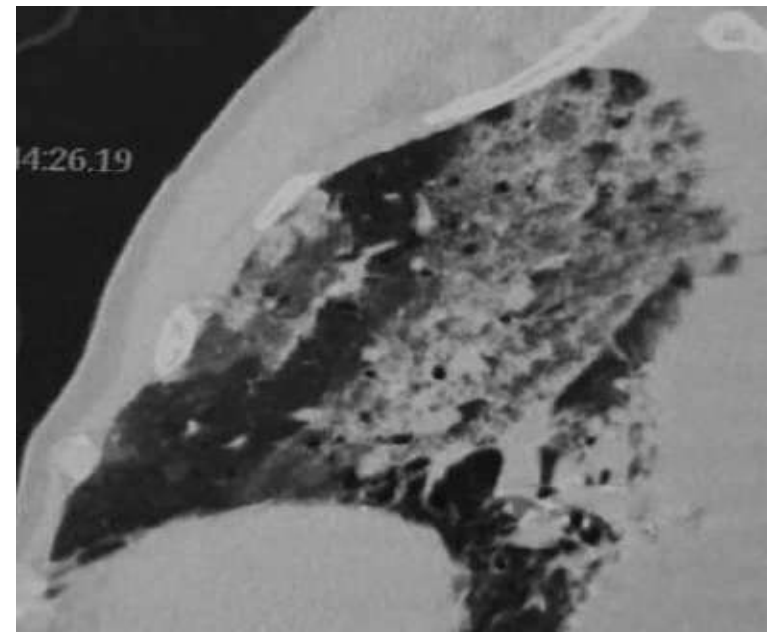

Figure 1b: Posterior predominant ground glass opacity.

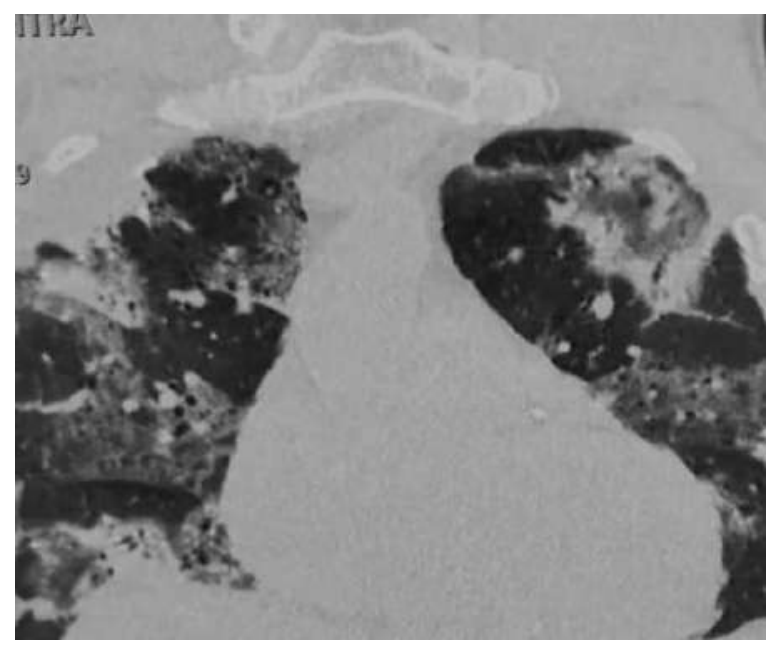

Figure 1c: Bilateral ground glass opacity with atoll sign at lul, i.e peripheral rim of consolidation around ground glass opacity

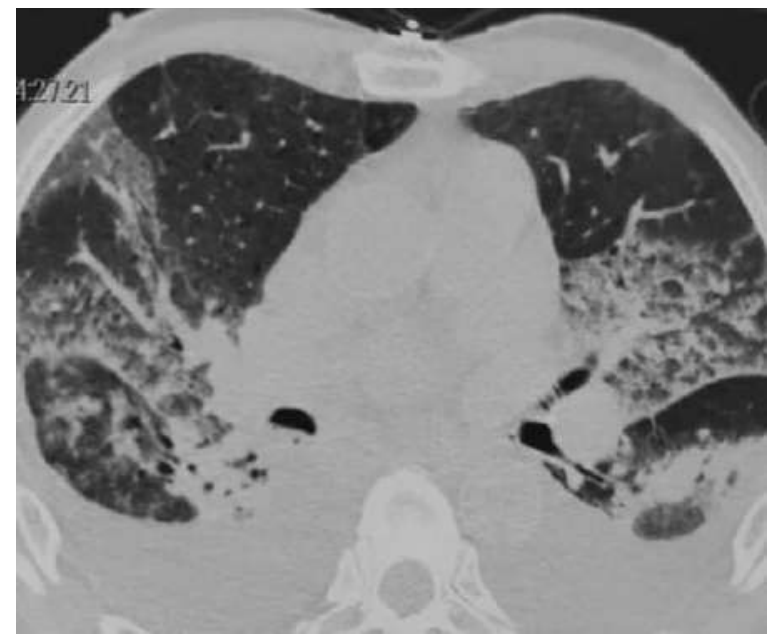

Figure 1d: Bilateral septal thickening

\section{HRCT Findings}

Extensive bilateral ground-glass opacities and airspace consolidation

Architectural distortion

Abnormalities predominantly basilar and dependent.

\section{End Stage IPF}

Idiopathic pulmonary fibrosis is more common in smokers and ex-smokers than in lifelong non-smokers, and more common in men than in women.IPF usually occurs in adult older than 55 yrs (median age of diagnosis, $66 \mathrm{yrs}$ ) and is rare in patients less than $50 \mathrm{yrs}$ of old. Patients who have IPF typically present with chronic exertional dysponea, cough, bibasilar inspiratory crackles and finger clubbing. Pulmonary function tests show a restrictive pattern with reduced lung volumes and impairment in gas excahge. IPF has a poor prognosis with a median survival of 2.5 to 3.5 yrs after the time of diagnosis. It is predominantly associated with asymmetric peripheral honeycombing and large lung cysts. Irregular reticular opacities are also noted which are predominantly sub-pleural in location.

Honeycomb cysts are air-filled, cystic spaces with fibrous walls lined by bronchiolar epithelium; predominantly in peripheral and sub-pleural location; with clearly definable walls $1-3 \mathrm{~mm}$ in thickness; occurring in multiple layer.

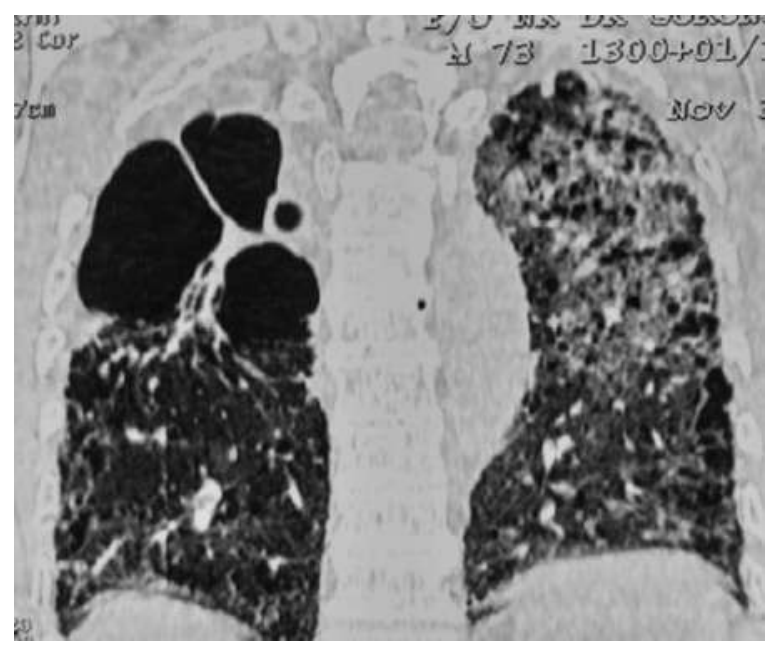

Figure 2a: Extensive honeycombing with para-septal emphysema and conglomerate fibrosis. 


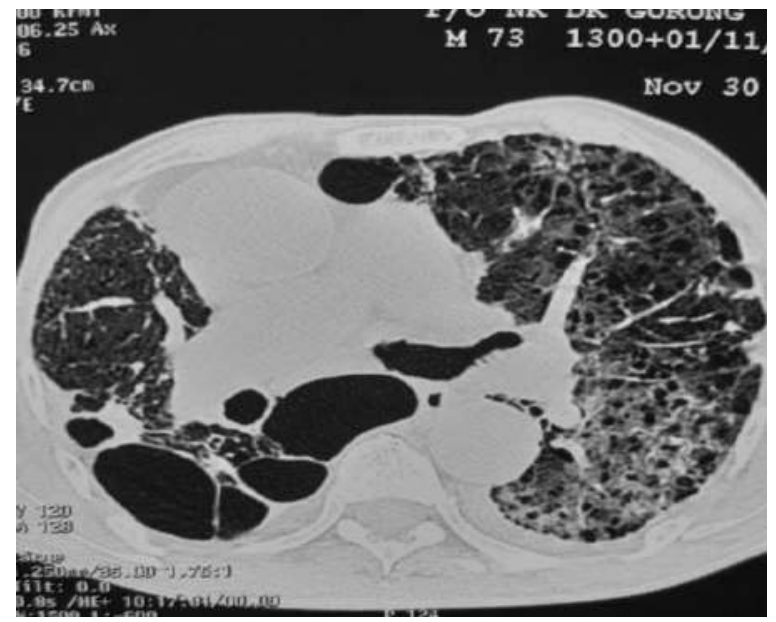

Figure 2b: Extensive reticulation with architectural distortion Sub -acute HP

Hypersensitivity pneumonitis (HP) is a diffuse granulomatous interstitial lung disease caused by an abnormal immunologic reaction to antigens contained in various organic dusts. There is an interstitial infiltrate comprising lymphocytes and plasma cells, with a propensity for small airways (bronchioles) involvement. Predictably, at CT there is diffuse ground glass opacification, ill-defined centri-lobular nodules and lobular areas of decreased attenuation on images performed at end-expiration (Hansell et al., 1996). A high index of suspicion and meticulous acquisition of an environmental and occupational history are essential in making the diagnosis. There is close similarity in CT appearance of sub-acute hypersensitivity pneumonitis with respiratory bronchiolitis -associated interstitial lung disease. However, consideration of the smoking history may help in differentiation: a history of smoking is the norm in the vast majority of patients with RB-ILD, where cigarette smoke appears to lead to a relative protection against the development of hypersensitivity pneumonitis.

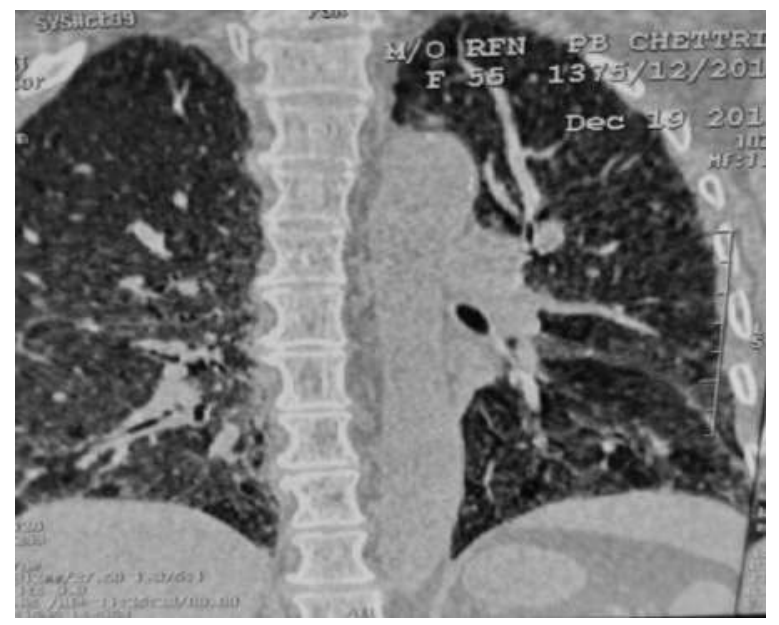

Figure 3a: Diffuse ground glass haze.

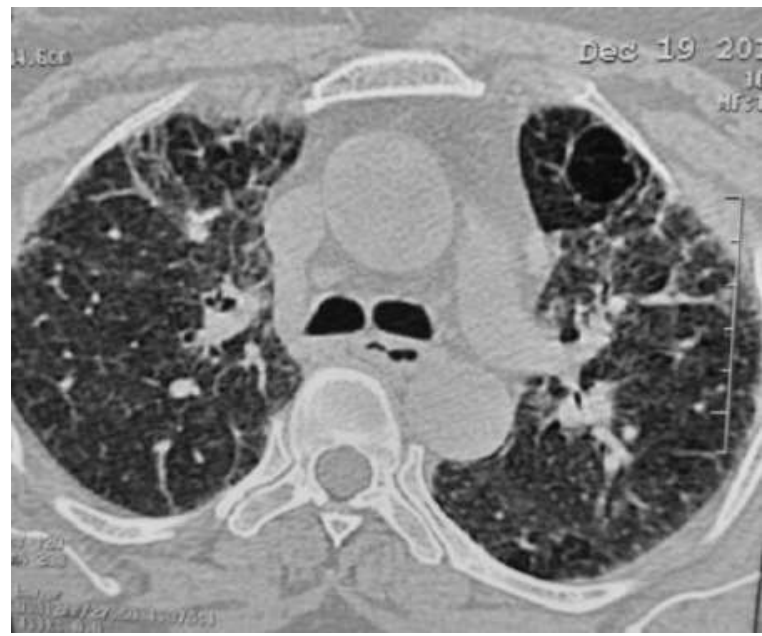

Figure 3b: Distinct lobular area of decreased attenuation/ mosaic attenuation with air cyst.

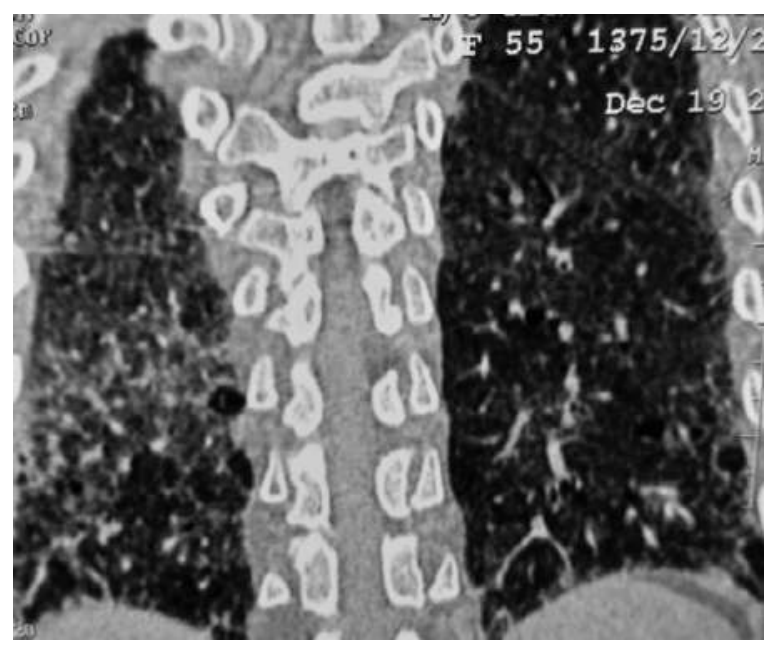

Figure 3c: Small sub-pleural reticulation.

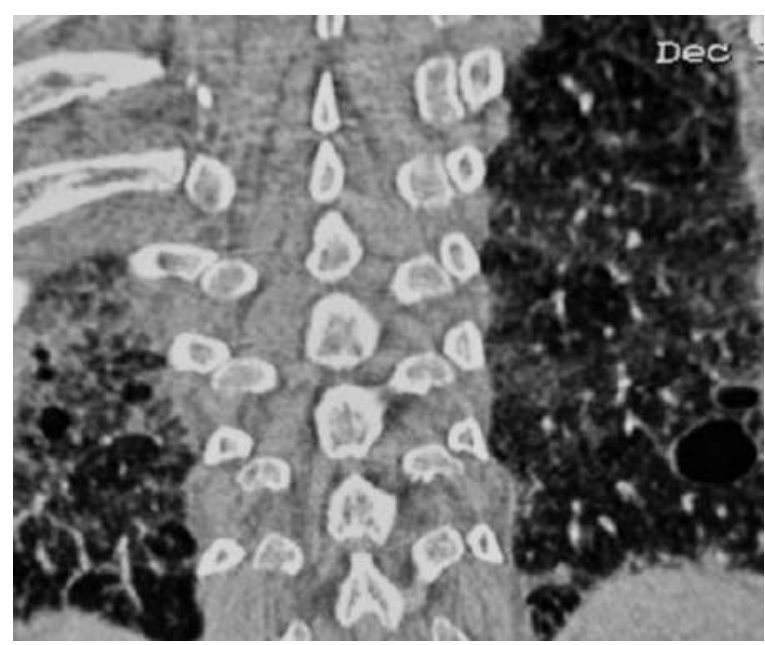

Figure 3d: Lobular area of air-trapping

\section{HRCT Findings}

Patchy or diffuse ground glass opacity superimposed on poorly defined small centri-lobular nodules. 
Lobular areas of decreased attenuation (mosaic perfusion).

Headcheese Sign (Combination of ground glass opacity and patchy mosaic perfusion)

Localised area of decreased attenuation and vascularity with striking lobular distribution.Areasof air-trapping on expiratory scan.

Diffuse, mid lung or upper lung predominance

\section{Lympahangitis Carcinomatosis}

Pulmonary lymphangitis carcinomatosis refers to tumour growth in the lymphatic system of the lung with a underlying background of primary carcinoma elsewhere in the body. It usually results from haematogenous spread to lung, with subsequent interstitial and lymphatic invasion, but can also occur because of direct lymphatic spread of tumor from mediastinal and hilar lymph nodes. Shortness of breath is typical.

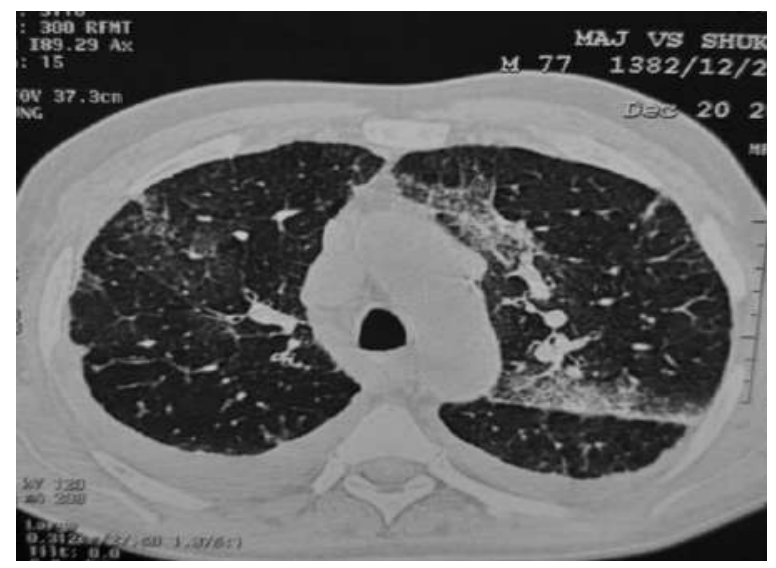

Figure 4a: There is beaded thickening of the left oblique fissure with asymmetrical interlobular septal thickening.

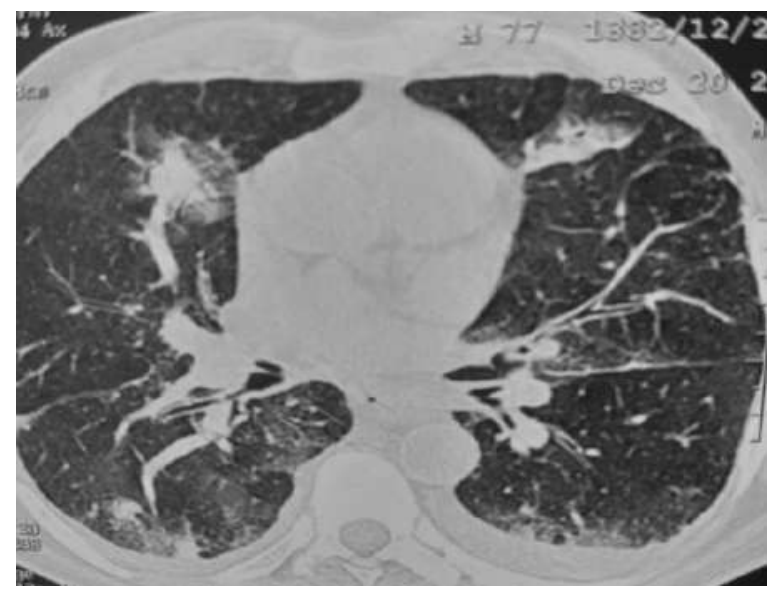

Figure 4b: Sub-pleural nodule on the right with bilateral pleural effusion and peri-bronchial consolidation.

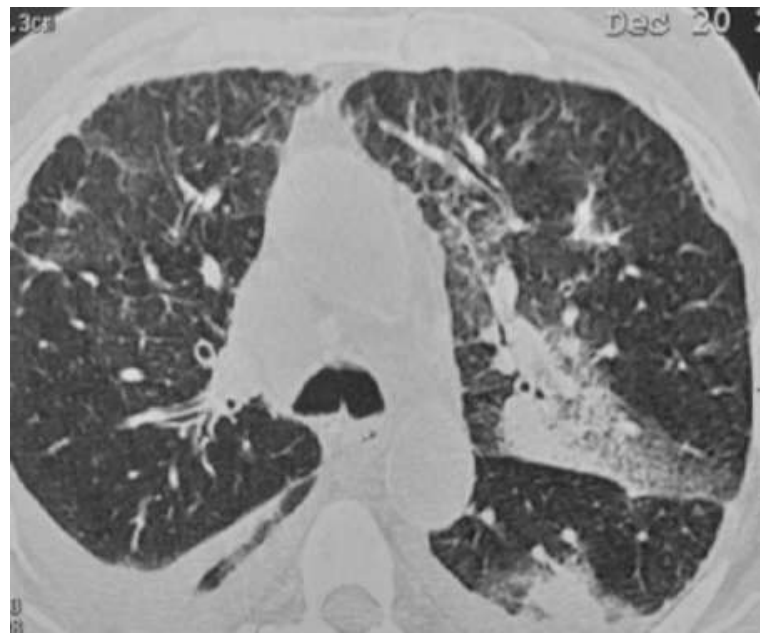

Figure 4c: Septal thickening on the left side.

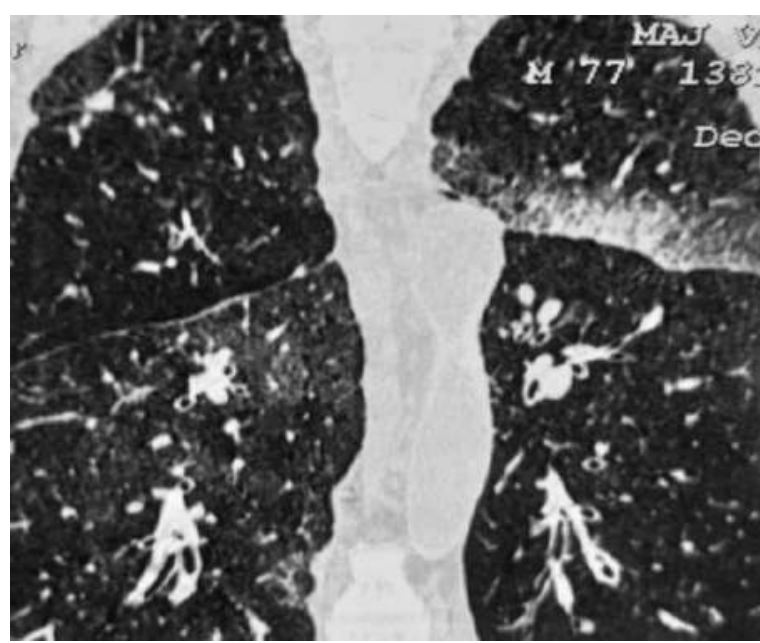

Figure 4d: Extensive fissural nodule. Patient has a known h/o Ca Rectum, presented with dyspnoea.

\section{HRCT Findings}

Smooth or nodular peri-bronchovascular interstitial thickening

Smooth or nodular interlobular septalandfissural thickening

Sub-pleural nodules

Normal lung architecture; no distortion

Diffuse patchy or unilateral distribution.

Lymph node enlargement

Pleural effusion

NSIP

Non-specific interstitial pneumonitis resembles UIP, but is associated with a significantly different course and outcome. The typical patient with NSIP is between 40 and 50 yrs old and is usually about a decade younger 
than the patient with IPF. It is associated with a better rate of survival. This pattern of IIP may be idiopathic but, more commonly, is seen in a variety of clinical contexts, including connective tissue disorder (especially systemic sclerosis) and as a consequence of drug related toxicity. At a histologic level, there is a temporally and spatially uniform appearance, which in stark contrast to what is seen in UIP where there is varying amounts of interstitial inflammation and fibrosis. In some patients, over time, the extent of ground glass may decrease and become replaced by reticulation (i.e with UIP-like features) (Silva et al., 2008).

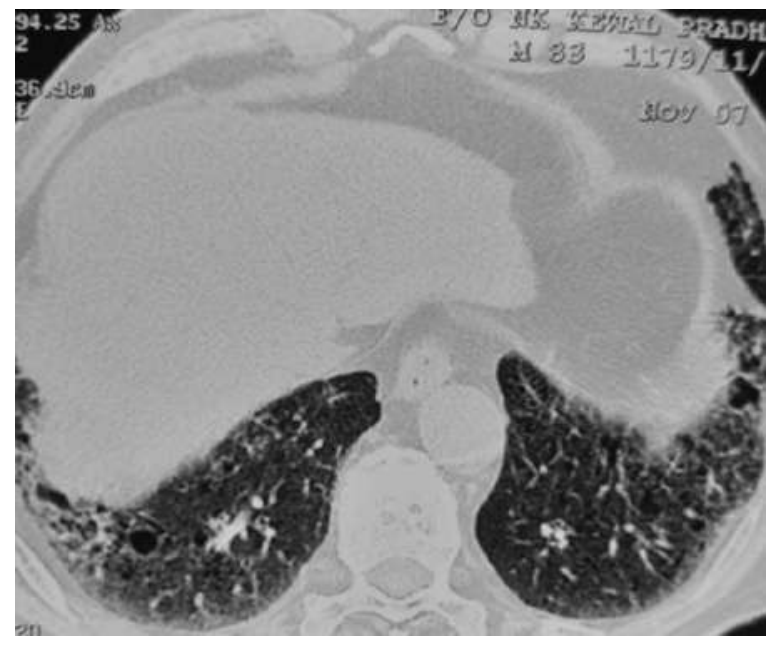

Figure 5a: Sub-pleural reticulation bilaterally.

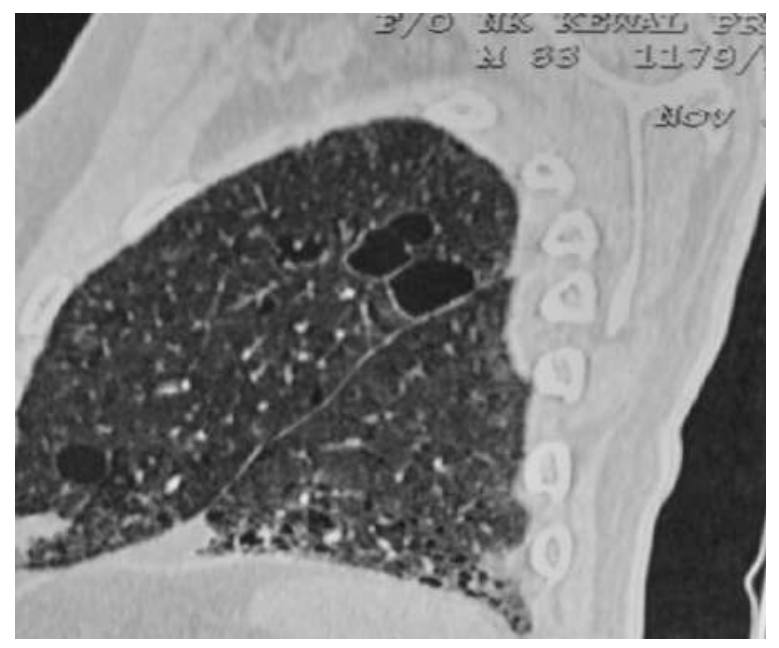

Figure 5b: Minimal honeycombing with lung cyst abutting the fissure.

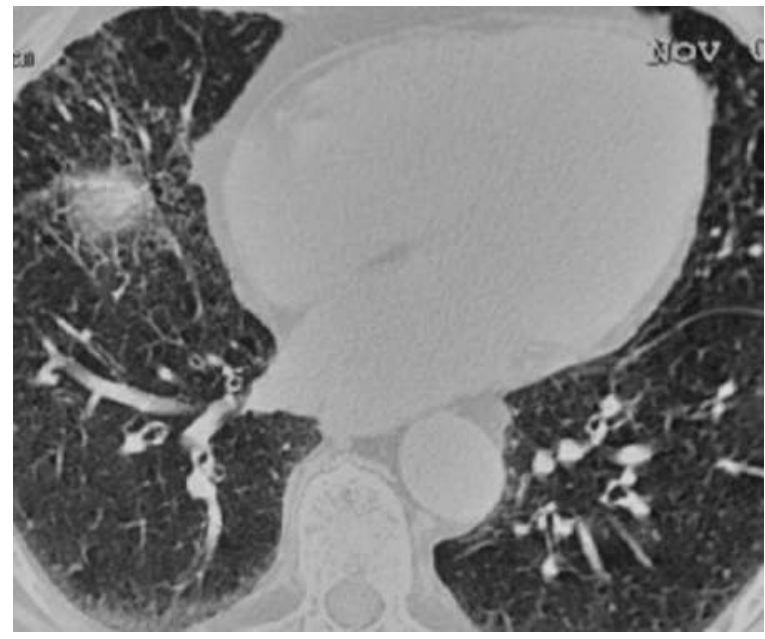

Figure 5c: Patchy ground glass opacity.

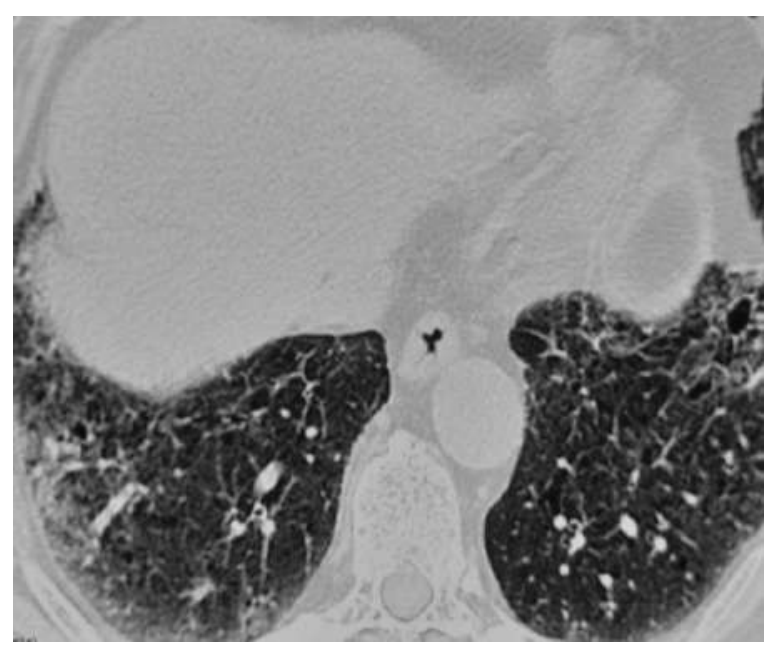

Figure 5d: Patchy septal thickening.

\section{HRCT Findings}

Patchy and peripheral ground-glass opacity, typically bilateral and symmetrically distributed in the lower zones.

Airspace consolidation

Fine reticular opacities

Traction bronchiectasis with fibrotic NSIP with relative sparing of the sup-pleural space in $43 \%{ }^{10}$.

Honeycombing (uncommon or minimal in extent) with fibrotic NSIP

\section{COP (Cryptogenic Organizing Pneumonia)}

Organizing pneumonia represents a histologic pattern characterized by loose plugs of connective tissue and granulation tissue in the air spaces and distal airways. Interstitial inflammation and fibrosis are usually minimal or absent. It is a common response to the lung injury and is the part of the normal process of lung repair. Organizing pneumonia was first recognized in 1923 as a 
response to unresolved pneumonia. Patients are typically middle aged and there is no preponderance of either sex. Frequently patient had a systemic illness lasting a few weeks or months with a non-productive cough, dysponea, malaise and a low grade fever. Often antibiotics have been administered without any response and no organism has been identified. The dominant finding in OP is bilateral consolidation that is peripheral often with sparing of the sub-pleural portion of the lung. Migration of the areas of consolidation and spontaneous regression of consolidation are helpful in narrowing the differential diagnosis to COP, eosinophilc pneumonia, pulmonary hemorrhage and pulmonary vasculitis.
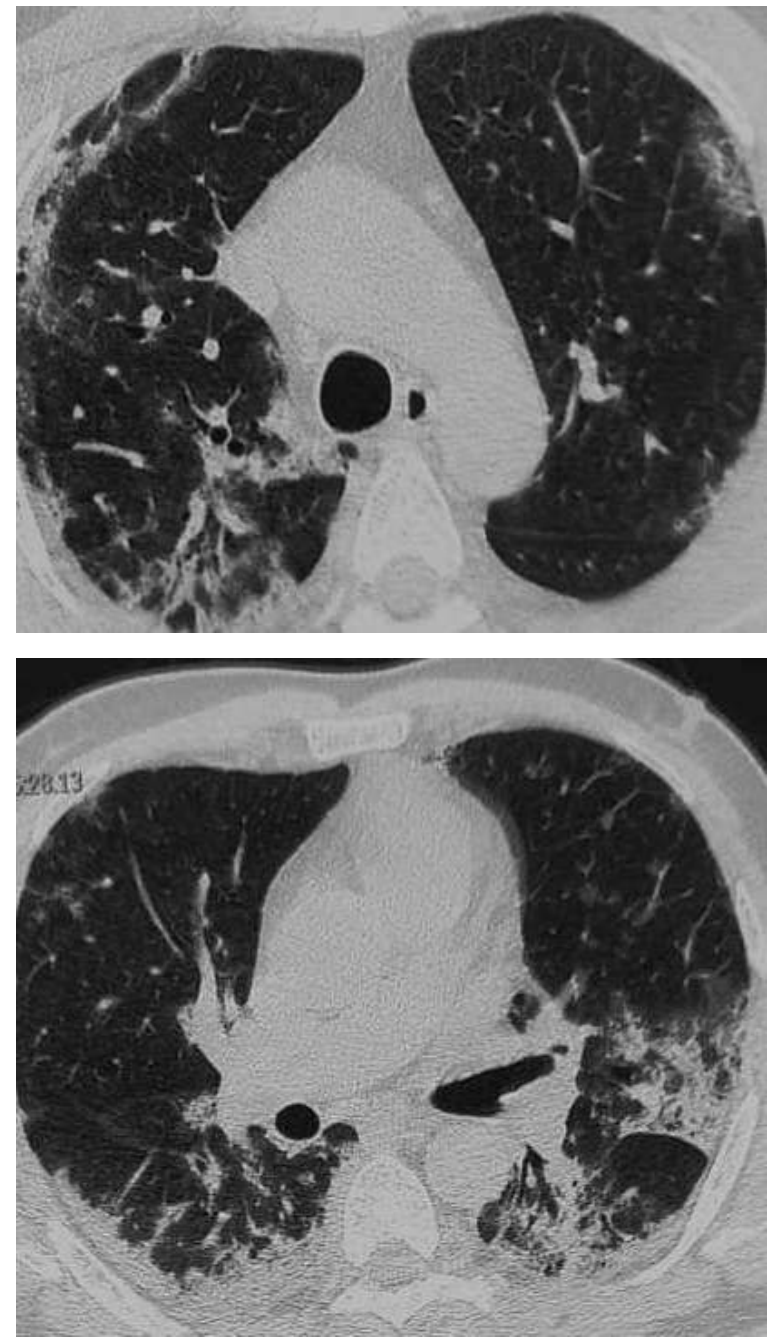

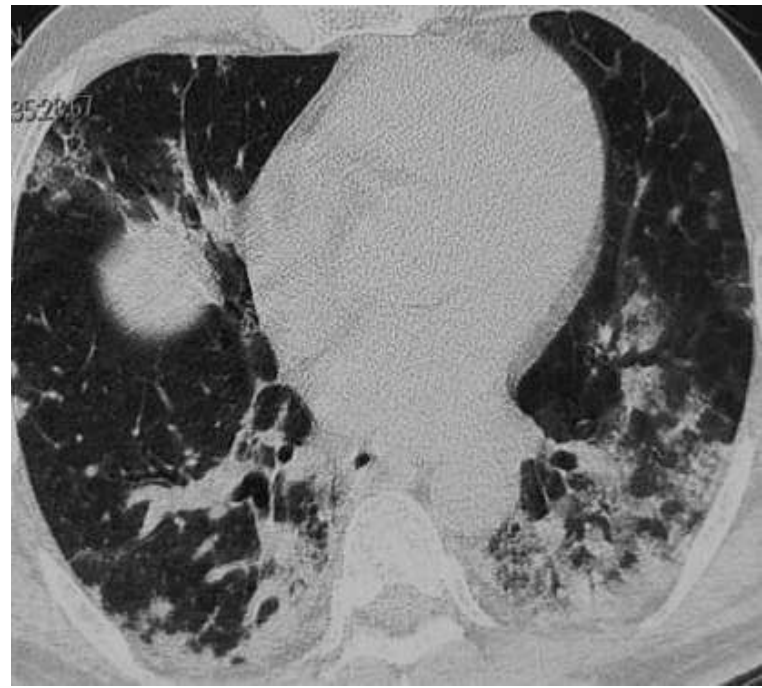

Figure 6a, 6c, 6d: Peripheral ground glass opacity with consolidation and fibrosis with an apico-basal gradient.

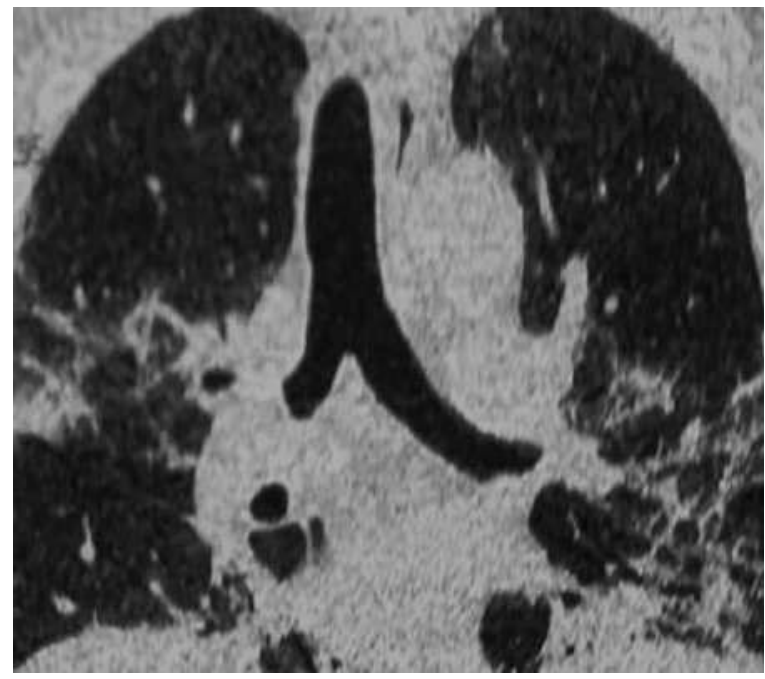

Figure 6b: Peri-lobular thickening

\section{HRCT Findings}

Characteristic peripheral or peri-bronchial patchy consolidation (sometimes with sub-pleural area involvement) also with air-bronchogram and mild cylindrical bronchial dilation.

Typically in mid and lower zones.

Increase of consolidation over several weeks after antibiotic therapy

Paucity or absence of reticular opacity with preserved lung volume.

Predominant peri-lobular pattern of involvement. These opacities are of greater thickness than those encountered in thickened inter-lobular septa and have an arcade of polygonal appearance. 
Patchy bilateral ground glass opacities. There may be illdefined centri-lobular nodules, usually randomly distributed in lungs.

Rarely amass or nodules that may cavitate or reproduce the typical appearance of an "atoll sign". It is due to the presence of a ring consolidation surrounding normal lung or ground glass opacification. It is considered relatively specific for OP as it is found in $20 \%$ patients with COP.

\section{Sarcoidosis}

Sarcoidosis is a common idiopathic granulomatous disease characterized by a peri-lymphatic distribution of nodules and lymphadenopathy early in its course. Clinical symptoms may be mild in patients with extensive abnormalities. Some patients develop extensive fibrosis. The basic abnormality in patients with sarcoidosis consists of small nodules, occurring in a perilymphatic distribution, and visible in relation to (a) the peri-broncho-vascular axial interstitium, that serves to support and invest the central, para-hilar vessels and bronchi, (b) the fissures (c) the costal sub-pleural regions (d) inter-lobular septaand (e) centri-lobularregion. Typically nodules predominate in relation to the peribroncho-vascular interstitium, in relation to pleural surfaces such as fissures and in the centri-lobular region.

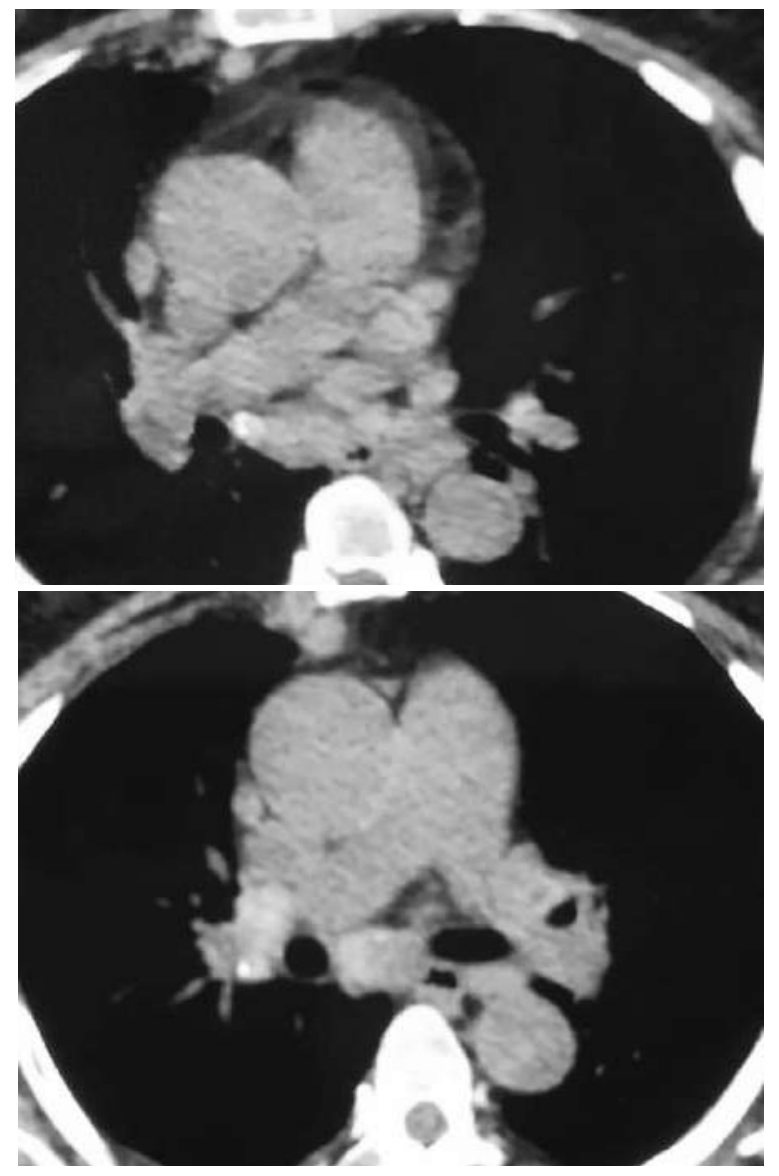

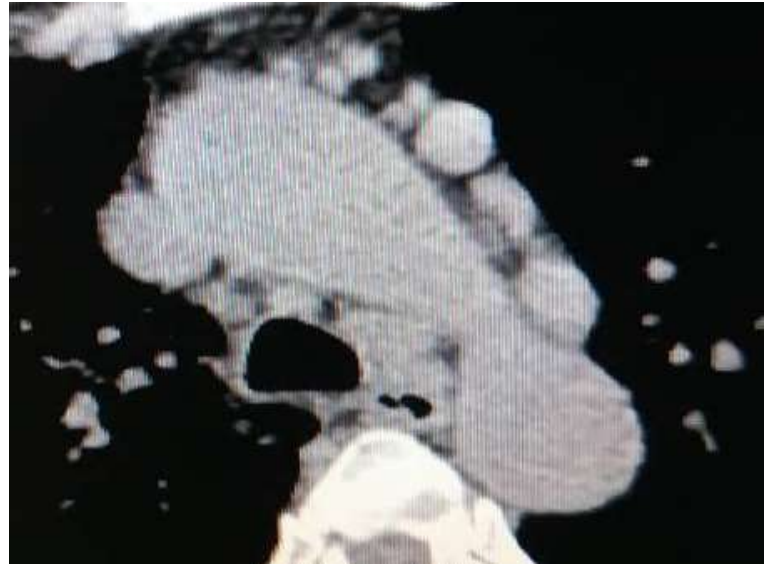

Figure 7a, 7b, 7c: Significantly enlarged mediastinal lymphnode.

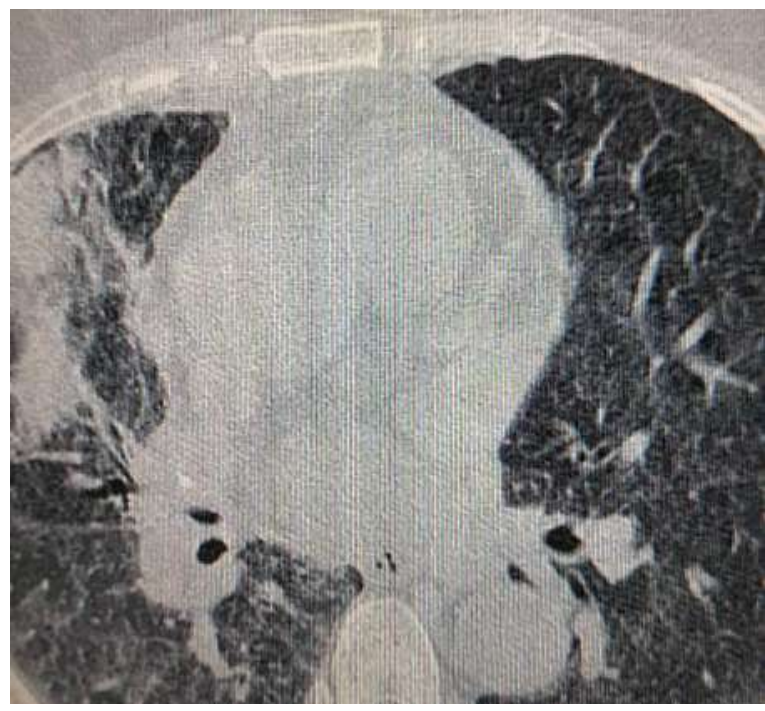

Figure 7d: Patchy upper lobar consolidation.

\section{HRCT Findings}

Smooth or nodular peri-bronchovascular interstitial thickening involving mid and upper lungs.

Small, well-defined sub-pleural nodules (fissures)

Centri-lobular nodules.

Coalescing granulomas form large mass like nodules (1-4 $\mathrm{cm})$, i.ealveolar sarcoid.

There may be ground-glass opacity

Lymph node enlargement, usually symmetric. Calcification often hazy or eggshell.

Extensive fibrosis and traction bronchiectasisin stage IV sarcoidosis.

\section{Pneumocystis Jiroveci Pneumonia}

Observed predominantly under the background of immune-suppression with AIDS with an insidious 
onset. However PCP in the non-AIDSimmunocompromised host is usually acute and manifested by an abrupt onset of fever, dry cough, dysponea and respiratory insufficiency.
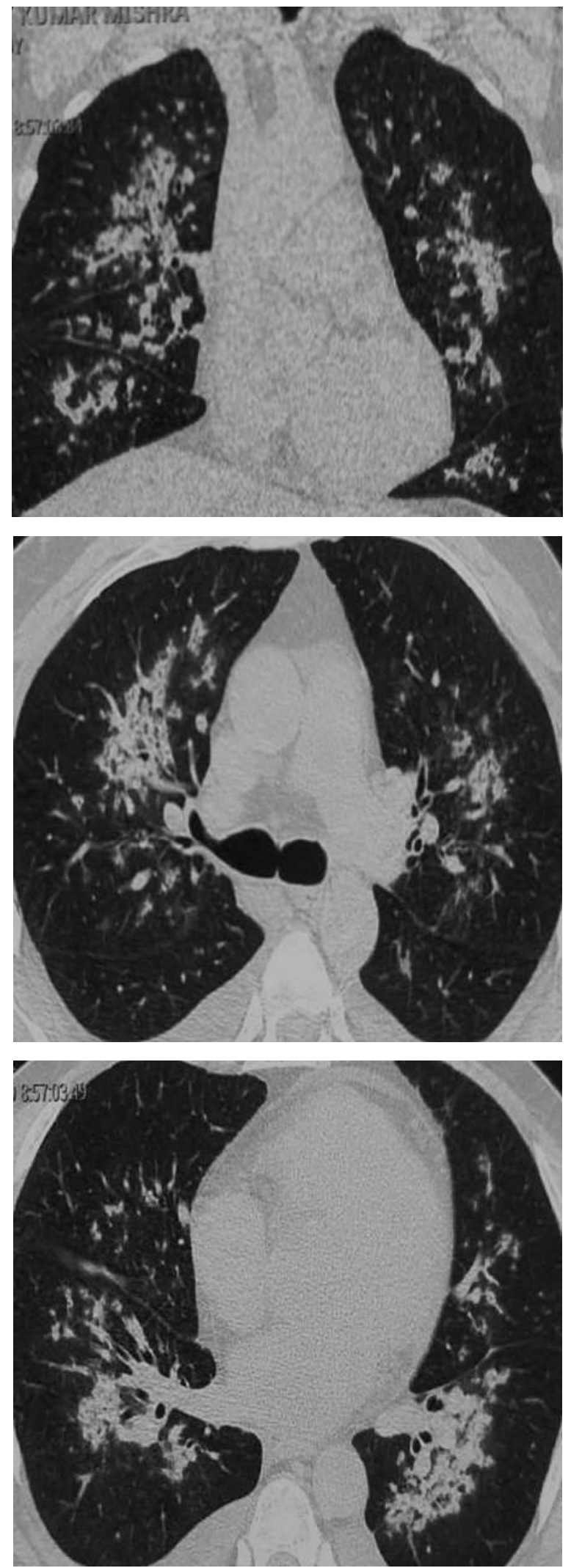

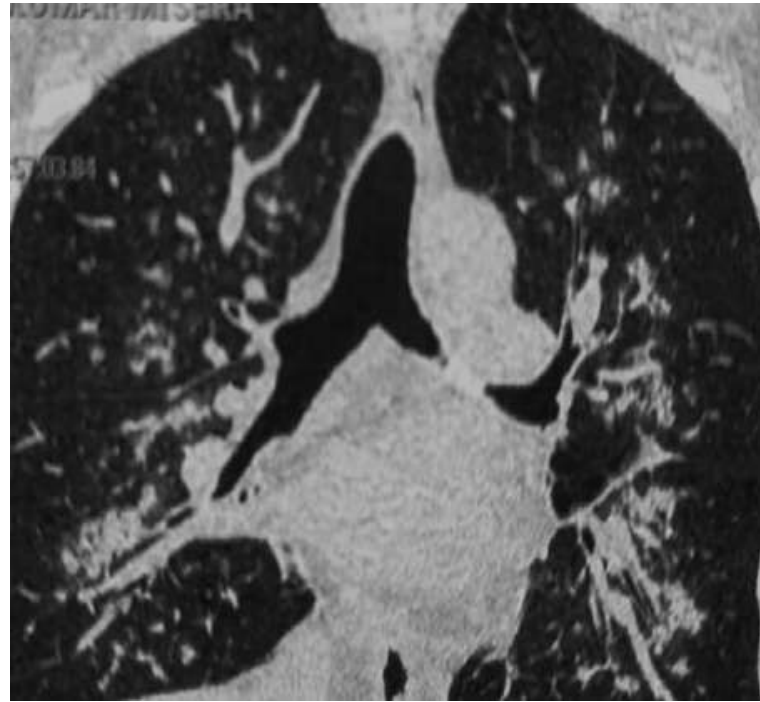

Figure 8a, 8b, 8c, 8d: Bilaterally symmetrical perihilar and peri-bronchial thickening with ground glass opacity. Relative preservation of lung architecture.

\section{HRCT Findings}

Patchy bilateral ground-glass opacity with predominantly peri-hilar distribution.

Reticular or inter-lobular septal thickening (resolving stage) with crazy-paving appearance.

Thick- or thin-walled, irregular, septatedcysts.

Centri-lobular opacities or tree in bud appearance is sometimes observed corresponding to infectious bronchiolitis.

\section{Alveolar Proteinosis}

Pulmonary alveolar proteinosis is characterized by filling of alveolar space with fine granular eosinophilic and periodic -acid Schiff -positive proteinaceous material, rich in lipid. Histologically this represents the intra-alveolar accumulation of large foamy macrophages .It occurs in association with a wide variety of disorder, including inhalation of silica, infection (eg. Pneumocystis jiroveci), andhematologic and lymphatic malignancies. In a study by Godwin et al., 1988 abnormalities ranged from ill-defined nodular opacities (air-space nodule) to large areas of ground glass opacity or confluent air-space consolidation. In many patients, areas of ground glass opacity or consolidation are sharply demarcated from the surrounding normal parenchyma, giving the abnormal areas a geographic appearance. The sharp margination of areas of lung opacity reflects lobular or lobular boundaries. 

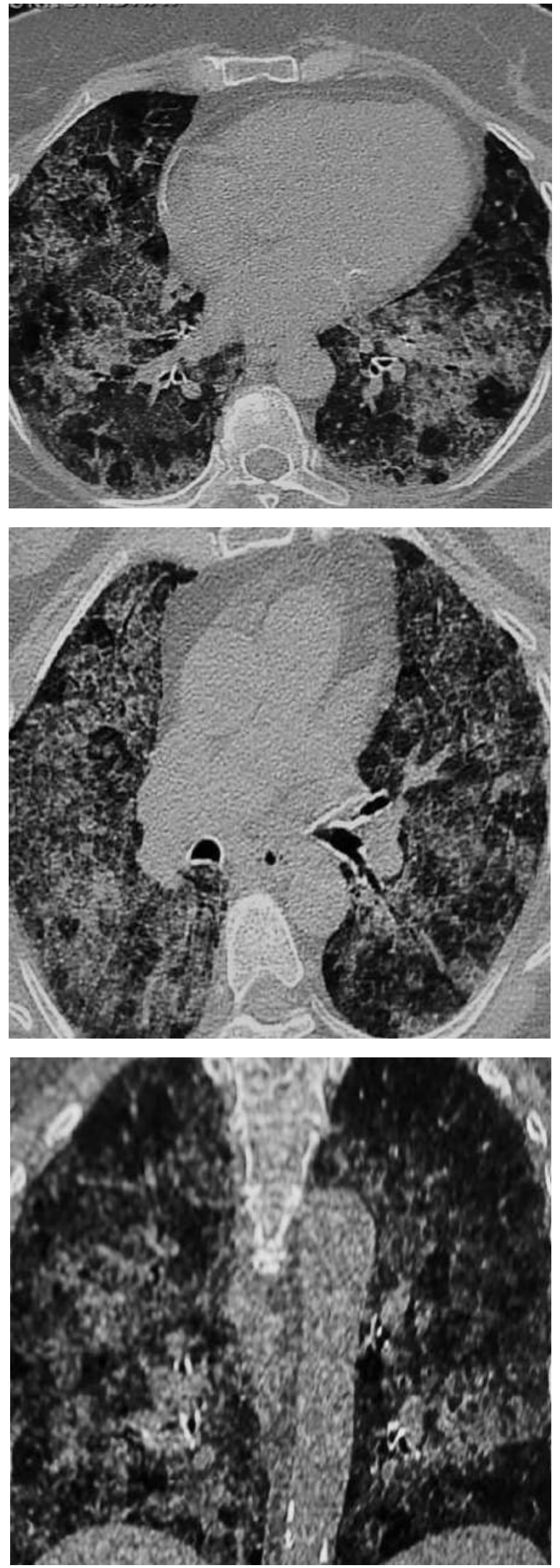

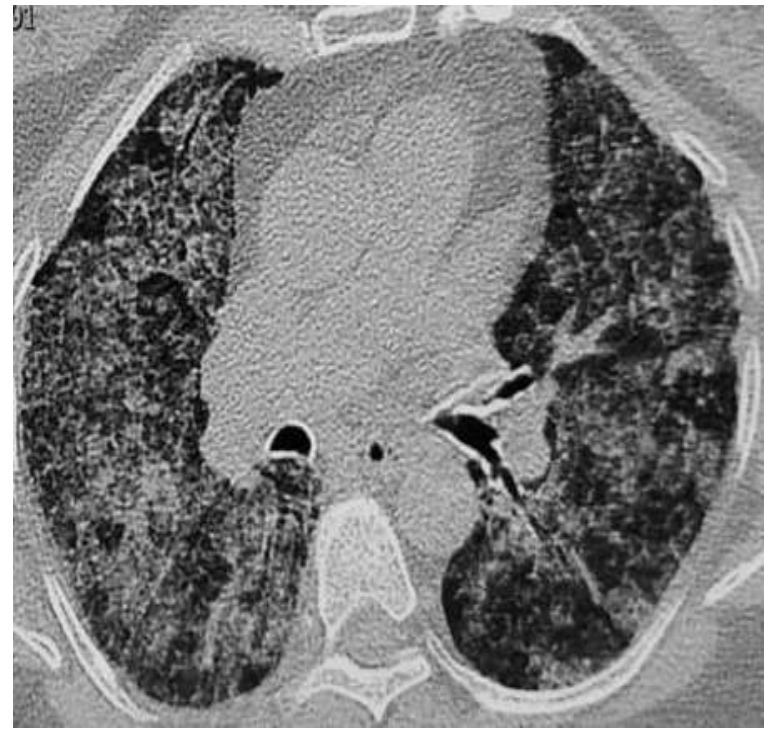

Figure 9a, 9b, 9c, 9d: Confluent area of ground glass opacity with septal thickening and with geographical distribution.

\section{HRCT Findings}

Patchy ground-glass opacity

Smooth septal thickening in abnormal areas

Crazy-paving.

Consolidation.

Patchy or geographicdistribution.

\section{CONCLUSION}

HRCT plays a pivotal role in the differential diagnosis of ILD. It relies on systematic analysis of the predominant CT pattern and distribution of lesions interpreted with the associated CT findings. The final diagnosis requires a combination of radiological, clinical and serological information, which is best accomplished with interdisciplinary cooperation.

\section{REFERENCES}

Desai S.R., Prosch H. and Galvin J.R., 2019. Plain film and HRCT Diagnosis of ILD. Disease of the Chest, Breast Heart and vessels, 2022: 37-45.

Fergusion E.C. and Berkowitz E.A., 2012. Lung CT: Part 2, The interstitial pneumonia-Clinical, Histologic and CT Manifestation. Am. J. Roentegenol, 199: W464-W476.

Godwin J.D., Muller N.L. and Takasugi J.E., 1988. Pulmonary alveolar proteinosis: CT Findings Radiology, 1988;169(3): 609-613. 
Gogali A. and Wells A.U., 2012. Diagnostic approach to interstitial lung disease. Curr. Respir Care Rep., 1: 199-207.

Hansell D.M., Wells A.U., Pandley S.P. and Muller N.L., 1996. Hypersensitivity pneumonitis: Correlation of individual CT patterns with functional abnormalities. Radiology, 199(1): 123-8.

Hansell D.M., Bankier A.A., Macmahon H., MaLoud T.C., Muller N.L. and Remy J., 2008. Fleischner Society: Glossary of terms for thoracic imaging. Radiology, 246: 697-722.

Hunninghake G.W., Zimmerman M.B., Schwartz D.A., King Jr. T.E., Lynch J., Hegele R., Waldron J., Colby T., Muller N., Lynch D., Galvin J., Gross B., Hogg J., Toews G., Helmers R., Cooper Jr. J.A., Baughman R., Strange C. and Millard M., 2001. Utility of a lung biopsy for the diagnosis of idiopathic pulmonary fibrosis. Am. J. Respir Crit. Care, 164: 193-6.
Primack S.L., Hartman T.E., Ikezoe J., Akira M., Sakatani M. and Muller N.L., 1993. Acute interstitial pneumonia: Radiographic and CT findings in nine patients. Radiology, 188(3); 817-820.

Silva C.I.S., Muller N.L., Hansell D.M., Lee Y.S., Nicholson A.G. and Wells A.U., 2008. Non specific interstitial pneumonia and Idiopathic pulmonary fibrosis: Changes in pattern and distribution of the disease over time. Radiology, 247(1): 251-9.

Ujita M., Renzoni E.A., Veeraraghavan S., Wells A.U. and Hansell D.M., 2004. Organizing Pneumonia: Peri-lobualr pattern at thin section CT Radiology, 232: 757-761.

Vourlekis J.S., Brown K.K., Cool C.D., Young D.A., Cherniack R.M., King T.E. and Schwarz M.I., 2000. Acute interstitial Pneumonitis. Case series and review of the literature, Medicine (Baltimore), 79(6): 369-378. 